\title{
A rare cause of respiratory distress in a newborn: Pneumomediastinum
}

\author{
Sinem GULCAN KERSIN (D), Hulya OZDEMIR (D), Hulya Selva BILGEN (D), Asli MEMISOGLU (D), Eren OZEK (D) \\ Division of Neonatology, Department of Pediatrics, School of Medicine, Marmara University, Istanbul, Turkey.
}

Corresponding Author: Sinem GULCAN KERSIN

E-mail: sinemgulcan@hotmail.com

Submitted: 26.08.2020 Accepted: 16.10 .2020

\begin{abstract}
Pneumomediastinum is an uncommon cause of neonatal respiratory distress and occurs in approximately 2.5 per 1000 live births. Although, the most common cause of pneumomediastinum is an underlying lung disease, it can be seen in newborns who undergo resuscitation during delivery without a predisposing factor. Initial diagnosis by chest X-ray can be difficult in some cases. The "spinnaker-sail sign" is an uncommon radiological appearance of pneumomediastinum. Careful conservative management can result with spontaneous resolution without long-term sequelae. In this case report, we present a neonate who developed pneumomediastinum. Keywords: Newborn, Birth trauma, Pneumomediastinum, Respiratory distress, Spinnaker-sail sign
\end{abstract}

\section{INTRODUCTION}

Pneumomediastinum is an uncommon cause of neonatal respiratory distress and occurs in approximately 2.5 per 1000 live births [1]. Spontaneous pneumomediastinum is usually caused by alveolar rupture resulting from a sudden increase in the thoracic pressure. Although, the most common cause of pneumomediastinum in children is an underlying lung disease, it can be seen in neonates who require resuscitation during delivery without a predisposing factor [2]. In this case report, we aimed to present a neonate who underwent resuscitation in the delivery room and developed pneumomediastinum.

\section{CASE REPORT}

A baby girl was born at 37 weeks of gestation with a birth weight of $3675 \mathrm{~g}$ to a 37-year-old multiparous mother by vaginal delivery. She required positive pressure ventilation by T-piece due to respiratory distress after delivery. The baby's $1^{\text {st }}$ and $5^{\text {th }}$ minute APGAR scores were 7 and 9 , respectively. Due to respiratory distress, the baby was admitted to the neonatal intensive care unit. The physical examination revealed caput succedaneum, edematous eyelids, petechiae, and ecchymosis around the eyes. Breath and heart sounds were decreased on the right hemithorax. Crepitation was palpated bilaterally on the neck and upper chest wall due to subcutaneous emphysema. Moro reflex was poor on the right side but the grasp reflex was present on the right hand. Due to grunting and tachypnea, she was intubated and placed on mechanical ventilation with minimal settings. Empiric antibiotics, ampicillin and gentamicin were commenced to cover the risk of sepsis due to respiratory distress. The laboratory test results were as follows, total leucocyte count: $12000 / \mathrm{m}^{3}$ hemoglobin: $15.1 \mathrm{gr} / \mathrm{dl}$, thrombocyte count: $149000 / \mathrm{m}^{3}$, C-reactive protein: $9.4 \mathrm{mg} / \mathrm{L}(0$ 5), procalcitonin: $19.6 \mu \mathrm{g} / \mathrm{L}(0-2)$; blood gas analysis, $\mathrm{pH}: 7.19$, $\mathrm{CO}_{2}$ : $58 \mathrm{mmHg}, \mathrm{HCO}_{3}: 21.6 \mathrm{mEq} / \mathrm{L}$, base excess: $8.2 \mathrm{mEq} / \mathrm{L}$, lactate: $2.3 \mathrm{mmol} / \mathrm{L}$. Pneumomediastinum was suspected due to a spinnaker-sail sign on the chest X-ray that was otherwise unremarkable (Figure 1).

A few hours after birth, the infant became hypotensive and dopamine infusion was started. Within 24 hours, the baby was clinically stabilized and her blood gases were within normal ranges. She was extubated at the end of the first day and was on $\mathrm{FiO}_{2}$ of $25 \%$. Her computed tomography (CT) findings showed pneumomediastinum without any mediastinal mass (Figure 2). Cranial and abdominal ultrasonographies were unremarkable. 
The image of pneumomediastinum persisted for three days and regressed spontaneously (Figure 3). She was consulted to physical therapy and orthopedics departments due to brachial

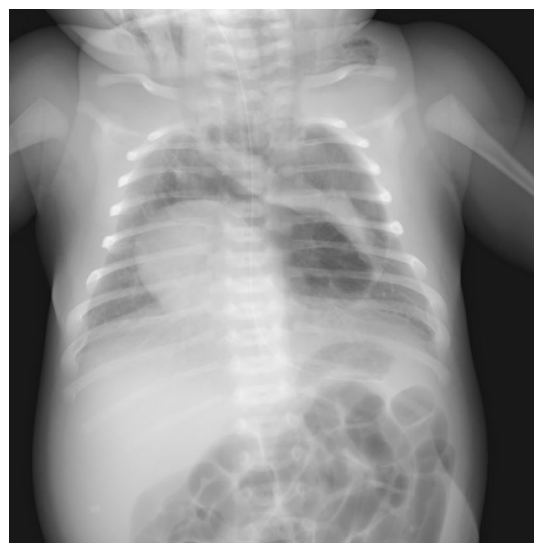

Figure 1. The chest $X$-ray on admission to Neonatal Intensive Care Unit

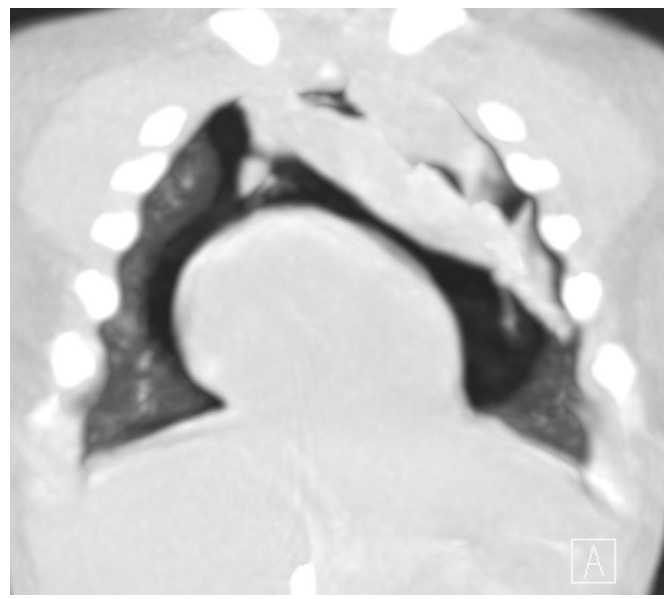

Figure 2. The CT scan of the patient on day 2

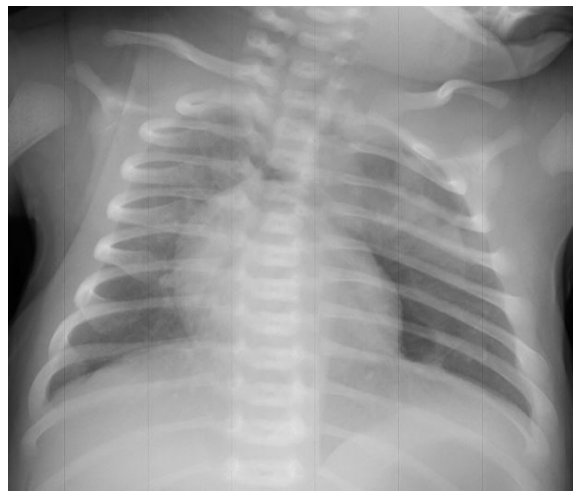

Figure 3. The chest X-ray before discharge plexus injury. The baby was discharged on the $7^{\text {th }}$ day of life. In the second month, her physical examination was totally normal including the chest X-ray (Figure 4).

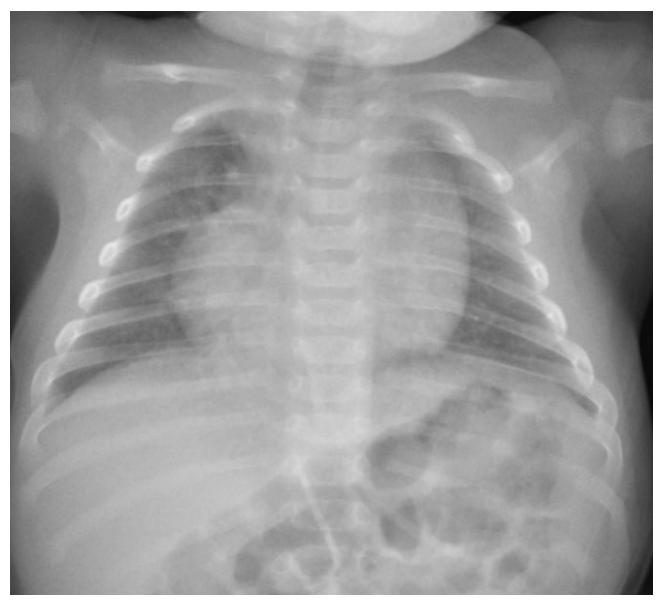

Figure 4. The chest X-ray at two months

Written informed consent was obtained from the family for publication of this case report and accompanying images.

\section{DISCUSSION}

Pneumomediastinum is a benign, self-limiting condition in neonates [3]. It occurs in approximately 2.5 per 1000 live births [1]. It occurs due to increases in alveolar pressure and air leakage to the mediastinum. Spontaneous pneumomediastinum has no association with mechanical ventilation for respiratory distress syndrome, tracheal or esophageal injury [4]. It is frequently associated with nonspecific birth-related trauma [5]. Most of the babies diagnosed with pneumomediastinum are asymptomatic at birth for that reason some studies reported lower incidences (1.7 per 1000 live births) [6,7]. Our case, who was symptomatic at birth, had signs of respiratory distress and required positive pressure ventilation by T-piece for 15 seconds. Additionally, she had brachial plexus injury.

Steele et al., evaluated chest X-rays of 550 babies within two hours after birth and diagnosed 13 pneumothorax and/or pneumomediastinum cases [1]. The incidence was higher (8\%) in intubated infants. The incidence of pneumomediastinum was detected in $2.3 \%$ and $1 \%$ in vaginal and caesarean section deliveries, respectively. According to their study, apparent predisposing or associated factors included intubation, congenital anomalies, and meconium-stained amniotic fluid while no significant correlation was found for prematurity, dysmaturity, small size for age, caesarean section, or hyaline membrane disease $[1,3]$.

The babies are usually diagnosed on plain chest $\mathrm{X}$ ray. The radiographic signs of pneumomediastinum include pneumopericardium, continuous diaphragm sign, continuous 
left diaphragm sign, Naclerio's V sign, and spinnaker-sail sign $[5,8]$. The "spinnaker-sail sign" might be accepted as an ominous sign in a term neonate, especially if it is accompanied by pneumopericardium and pneumoperitoneum [9]. This uncommon sign, where the thymic lobes are displaced superiorly and laterally by pneumomediastinum should not be confused with the thymic sail sign which is a normal finding in infants [10]. The "spinnaker-sail sign" was detected on the chest $\mathrm{X}$-ray in some cases who required bag valve mask ventilation due to continued dyspnea similar to our case who also required resuscitation by T-piece $[5,7]$.

The diagnosis can be difficult in some cases. Chest X-ray can be negative in up to $30 \%$ of cases [11]. For the exclusion of mediastinal masses, a CT scan can be used [7]. In our patient's chest X-ray, the typical "spinnaker-sail sign" due to the elevation of the thymus confirmed the diagnosis of pneumomediastinum. The presence of any mediastinal mass was ruled out by CT.

Pneumomediastinum resolves with supportive conservative treatment, with or without oxygen therapy, in most of the cases [3]. Similar to the cases reported in the literature, our case had a spontaneous resolution [7]. Deterioration of the clinical status due to pneumothorax, subcutaneous interstitial emphysema, and the need for drainage and mechanical ventilation had been reported in severe cases [3,9].

Pneumomediastinum is a rare cause of respiratory distress and initial diagnosis by chest X-ray can be difficult in some cases. Timely diagnosis of spontaneous pneumomediastinum is important. Pneumomediastinum should be considered especially in patients with respiratory distress who underwent resuscitation after a traumatic delivery. The "spinnakersail sign" is an uncommon radiological appearance of pneumomediastinum. Careful conservative management can result in spontaneous resolution without long-term sequelae.

\section{Compliance with the Ethical Standards}

Written informed consent was obtained from the family for publication of this case report and accompanying images.

Financial Support: The authors have no relevant financial information to disclose.

Conflict of Interest: The authors have no potential conflicts to disclose.

Author Contributions: SGK conceived the case report, drafted the manuscript and carried out the literature search. HO and SGK prepared the figures, have expert knowledge in this area and made critical revisions. $\mathrm{AM}, \mathrm{HB}$, and $\mathrm{EO}$ have expert knowledge in this area and made critical revisions. All authors read and approved the final manuscript.

\section{REFERENCES}

[1] Steele RW, Metz JR, Bass JW, DuBois JJ. Pneumothorax and pneumomediastinum in the newborn. Radiology 1971; 98: 629-32. doi: 10.1148/98.3.629.

[2] Versteegh FG, Broeders IA. Spontaneous pneumomediastinum in children. Eur J Pediatr 1991; 150: 304-7. doi: 10.1007/ bf01955926.

[3] Rocha G, Guimaraes H. Spontaneous pneumomediastinum in a term neonate - case report. Clin Case Rep 2018; 6: 314-6. doi: $10.1002 /$ ccr3.1352.

[4] Ammari AN, Jen A, Towers H, Haddad JJr, Wung JT, Berdon WE. Subcutaneous emphysema and pneumomediastinum as presenting manifestations of neonatal tracheal injury. J Perinatol 2002; 22: 499-501. doi: 10.1038/sj.jp.7210758.

[5] Lawal TA, Gluer S, Reismann M, Dordelmann M, Schirg E, Ure B. Spontaneous neonatal pneumomediastinum: the "spinnaker sail" sign. Eur J Pediatr Surg 2009; 19: 50-2. doi: 10.1055/s-2008.103.8393.

[6] Zuppa AA, D’Andrea V, Verrillo G, et al. Spontaneous neonatal pneumomediastinum: radiological or clinical diagnosis? J Obstet Gynaecol 2014; 34: 138-40. doi: 10.3109/01443.615.2013.830597.

[7] Teo SSS, Priyadarshi A, Browning Carmo K. Sail sign in neonatal pneumomediastinum: a case report. BMC Pediatr 2019; 19: 38. doi: 10.1186/s12887-019-1397-7.

[8] Bejvan SM, Godwin JD. Pneumomediastinum: old signs and new signs. AJR Am J Roentgenol 1996; 166: 1041-8. doi: 10.2214/ajr.166.5.8615238.

[9] Hwang J, Park H, Jun S, Lee HJ. Spinnaker sail sign accompanied with pneumopericardium and pneumoperitoneum. J Neonatal Biol 2015; 4: 2. doi:10.4172/2167-0987.100.0177.

[10] Sunilkumar M. Sail sign, significance in paediatrics and review of literature. J Res Med Dent Sci. 2014; 2: 86. doi: 10.5455/ jrmds.20142417.

[11] Jariwala PS, Kalaniti K, Wonko N, Daspal S, Mugarab Samedi V. The value of different radiological modalities in assessment of spontaneous pneumomediastinum: Case review and diagnostic perspective. AJP Rep 2019; 9: 72-5. doi:10.1055/s-0039.168.3862 